\title{
Epidemiology of Childhood Mental Illness: A Review of U.S. Surveillance Data and the Literature
}

\author{
David S. Younger ${ }^{1,2}$ \\ ${ }^{1}$ Department of Neurology, Division of Neuroepidemiology, New York University Langone Medical Center, New York, USA \\ ${ }^{2}$ College of Global Public Health, New York University, New York, USA \\ Email: david.younger@nyumc.org
}

How to cite this paper: Younger, D.S. (2017) Epidemiology of Childhood Mental Illness: A Review of U.S. Surveillance Data and the Literature. World Journal of Neuroscience, 7, 48-54. https://doi.org/10.4236/wjns.2017.71005

Received: January 3, 2017

Accepted: January 19, 2017

Published: January 22, 2017

Copyright $\odot 2017$ by author and Scientific Research Publishing Inc. This work is licensed under the Creative Commons Attribution International License (CC BY 4.0).

http://creativecommons.org/licenses/by/4.0/

c) (†) Open Access

\begin{abstract}
Background: Childhood mental illness is a major factor of overall lifespan of children continuing into adulthood. Population based surveys and public health surveillance provide the much needed evidence to understand mental health promotion, prevention, and treatment of mental illness in children in the United States. Objective: To review available current national survey data regarding mental illness in children over the past decade and to review the global implications of childhood mental illness. Results: Altogether, 13\% $20 \%$ of children experienced a mental health disorder from 1994 to 2011 . The prevalence of depression among children of $3-17$ years old was $3 \%$ and the prevalence of lifetime and past year major depressive episode were $12.8 \%$ and $8.1 \%$ respectively among adolescents of $12-17$ years old, with a prevalence of depression in the preceding 2 weeks of $6.7 \% .7 .1 \%$ of children of $12-17$ years old ever had a diagnosis of depression, 3.5\% had current depression and 5.1\% had a diagnosis of depression in the past year. Overall, $28.5 \%$ of high school students of 14 - 18 years old reported feeling so sad or hopeless every day for two weeks or more in a row that they stopped doing usual activities, higher among girls (35.9\%) than boys (21.5\%), and greater in Hispanic students (32.6\%) than white non-Hispanic $(27.2 \%)$ or black non-Hispanic students (24.7\%). The overall rate of suicide in children in 2010 was 4.5 per 100,000. Conclusions: Mental illness presents serious departures from the expected cognitive, social, and emotional development in children, and an important area for prevention in the U.S. and globally. Mental illness will be managed effectively when there is adequate access to treatment services to reduce its associated morbidity and mortality.
\end{abstract}

\section{Keywords}

Childhood, Mental Health, Epidemiology, Public Health 


\section{Introduction}

Mental illness refers to all diagnosed mental disorders characterized by sustained, abnormal alterations in thinking, mood, or behavior associated with distress and impaired functioning [1] [2]. According to the World Health Organization (WHO), mental illness accounts for more disability in developed countries than any other disorders including cancer and heart disease [3]. Its impact ranges from minor to severe disruptions in daily functioning with significant to incapacitating departures from anticipated cognitive, social, and emotional development. Mental illness is associated with a life-long risk of anxiety, depression and suicide in children [4]. Surveillance studies of mental illness are necessary to provide a more complete picture of the prevalence of mental disorders among children and to promote mental health. This study was performed to assess recent surveillance and survey information of the domestic and global epidemiology of childhood mental illness over the past decade using standard case definitions of mental disorders among school age children. Data regarding psychotic illness were not available for review.

\section{Methods}

Perou and colleagues [4] reviewed mental health surveillance in children that involves the ongoing and systematic collection, analysis, interpretation, and dissemination of data used in public health in populations. The derived data are essential to the public health goals of reducing the incidence, prevalence, severity, and economic impact of mental illnesses. Public health officials, academicians, health-care providers, and advocacy groups track trends in mental illness prevalence and severity. Younger [1] reviews the surveys and reporting systemic summarized below in detail.

\subsection{Surveillance Surveys}

The National Health Intervention Survey (NHIS) is administered by the National Center for Health Statistics on the health of the civilian noninstitutionalized U.S. population. It monitors the health of the U.S. population through the collection and analysis of data on a broad range of health topics by in-person household interviews. About 40,000 households per year were interviewed as of 2010.

The National Health and Nutrition Examination Survey (NHANES) employed by the National Center for Health Statistics accesses the health and nutritional status of children in the U.S. It collects information derived from interviews, physical examinations, laboratory tests, nutritional assessment, and DNA repositories. As of 2008, approximately 5000 persons per year were interviewed.

The National Vital Statistics System (NVSS) assembles mortality statistics from death certificates filed in the U.S. and processed by the Centers for Disease Control and Prevention (CDC).

The National Violent Death Reporting System (NVDRS) is a populationbased active surveillance system used by the CDC to provide a census of violent 
deaths in participating states, including child maltreatment deaths, intimate partner homicides, and suicides, and legal intervention deaths.

The National Survey on Drug Use and Health (NSDUH) is a primary source of statistical information of alcohol, tobacco, illicit drug and non-medical use of prescription drugs in the U.S. derived from in-person interviews of representative samples of the non-institutionalized population.

The Autism and Developmental Disabilities Monitoring (ADDM) network is a surveillance system conducted by the CDC that estimates the prevalence of Autism Spectrum Disorders (ASD) among 14 population-based sites from health and educational records of health providers and schools.

The National Survey of Children's Health (NSCH) is a cross-sectional, random-digit population-based telephone survey that collects information on the physical and emotional health of noninstitutionalized children age 17 years or under to provide state and national estimates of child health.

The School-Associated Violent Death Surveillance Study (SAVD) is administered by the CDC in collaboration with the U.S. Department of Education and the Department of Justice to describe the epidemiology of school-associated violent deaths and potential risk factors thereof.

The National Youth Risk Behavior Survey (YRBS) employs a three-stage cluster design to produce representative samples of health-risk behaviors in public and private high school students grades 9 - 12 that contribute to leading causes of death, disability, and social problems.

\subsection{Diagnostic Classification}

Surveillance survey tools estimate the prevalence and trends in mental illness using symptom patterns. The diagnostic terminology used to describe mental illness diagnostic categories may vary. The American Psychiatric Association recognizes diagnostic categories based on symptoms observed by a health professional or reported by the patient and classified mental disorders that are published in the Diagnostic and Statistical Manual of Mental Disorders, Fourth Edition, Text Revision (DSM-IV-TR) [5]. The WHO International Classification of Diseases, 10th Revision, Clinical Modification (ICD-10-CM) [6] has defined mental illness categories that are congruent but not identical to those in the DSM-IV-TR.

Childhood mental disorders include several categories some of which are primary mental illnesses while others have a close association with a mental disorder [1]. Attention deficit hyperactivity disorder (ADHD), oppositional defiant disorder (ODD) and conduct disorder frequently occur together characterized by developmentally inappropriate levels of inattention, hyperactivity, impulsivity, or a combination thereof which impairs functioning in multiple setting. The ASD are a group of neurodevelopmental disorders characterized by impairments in social interactions and communications as well as restricted repetitive and stereotypes patterns of behavior that emerge in the first few years of life. Mood and anxiety disorders are characterized by feelings of depression, exaggerated 
anxiety or fear, low self-esteem, or all of them that persist or repeat over period of months or years. Substance use disorders and substance use refers to the use of alcohol and illicit drugs such as marijuana and inhalants which among children have social, financial and health consequences. Tic disorders include chronic motor or chronic vocal tics, transient tic disorder, and Tourette Syndrome (TS), the latter characterized by persistent motor and vocal tics that least for a least a year. While not a primary mental disorder, TS may have mental illness as an associated feature.

\section{Results}

The prevalence of depression among children age 3 - 17 years was $3 \%$ according to the NSCH whereas the NSDUH and NHANES estimated the prevalence of lifetime and past year major depressive episode (MDE) to be $12.8 \%$ and $8.1 \%$ respectively among adolescents age $12-17$ years, with a prevalence of depression in the preceding 2 weeks of $6.7 \%$. According to the NHIS, $7.1 \%$ of children age 12 - 17 years ever had a diagnosis of depression, 3.5\% had current depression and $5.1 \%$ for had a diagnosis of depression in the past year. The YRBS reported that $28.5 \%$ of high school students age $14-18$ years reported feeling so sad or hopeless every day for two weeks or more in a row that they stopped doing usual activities, higher among girls (35.9\%) than boys (21.5\%), and greater in Hispanic students $(32.6 \%)$ than white non-Hispanic $(27.2 \%)$ or black non-Hispanic students (24.7\%).

Parent-reported anxiety among children in the U.S. estimated by the NSCH that focused primarily on phobias in children age $2-17$ years noted a prevalence of $4.7 \%$ of ever having anxiety, and a $3 \%$ prevalence of current anxiety disorders. NHANES noted a prevalence of self-reported generalized anxiety disorder of $0.7 \%$, and phobias and fears in $2.6 \%$. NHANES estimated that $8.3 \%$ of self-reported adolescents age $12-17$ years had 14 or more mentally unhealthy days in the preceding month as a marker of mental distress.

Data on suicide gathered from the NVSS and NVDRS among 16 states noted an overall suicide rate among children age 10 - 19 years of 4.5 per 100,000 (NVSS), with 4.2 suicides per 100,000 between (NVDRS). White non-Hispanic children and non-Hispanic children had higher rates of suicide than black nonHispanic and Hispanic children. The commonest modes of self-injury were hanging, suffocation, and firearms. Among suicides reported by the NVDRS, $29.5 \%$ disclosed an intent to die by suicide before the act; while $35.5 \%$ had a diagnosed mental disorder at the time of death; and $26.4 \%$ were under treatment for a current mental disorder at the time of death. Overall, $21 \%$ had made a previous suicide attempt.

\section{Discussion}

Mental health disorders are substantial public health concerns because of their prevalence, early onset, impact and associated costs to the child, family and community. CDC-sponsored surveys such as NHANES and NHIS are useful in 
the development of national policies and in tracking the progress toward national health goals for children. The BRFSS survey provided data at state and local levels useful in planning. About $13 \%$ to $20 \%$ of children living in the U.S. experienced a mental disorder in a given year from 1994-2011 [4]. Based on self-reporting by the NSDUH, the prevalence of lifetime and past year MDE among adolescents from $2010-2011$ was $12.8 \%$ and $8.1 \%$ respectively. Parent reporting data from NSCH and NHIS noted a prevalence of ever having a diagnosed depression of $7.1 \%$ among adolescents age $12-17$ years. The prevalence of unhealthy days for two or more weeks in the past month was $6.7 \%$ according to NHANES, with $28.5 \%$ of high school students age $14-18$ years self-reporting sadness or hopelessness almost every day for two weeks or more causing them to stop usual activities (YRBS). Depression was higher among adolescent girls, and higher among Hispanic than white non-Hispanic or black non-Hispanic students.

Parent-reported anxiety among children age 2 - 17 years was $4.7 \%$ for ever being anxious and 3\% for a current anxiety disorder. Self-reported mentally unhealthy days dichotomized at 2 or more weeks in the past month was a useful indicator for the severity of anxiety and depressive disorders so noted in $8.3 \%$ of children overall. The overall suicide rate for persons aged 10 to 19 years old was 4.5 per 100,000 persons. Adolescent boys age 12 to 17 years were more likely than girls to suffer suicide, similarly, white non-Hispanic children and nonHispanic children of other races than black non-Hispanic and Hispanic children.

\subsection{Mental Illness and Millennium Development Goals}

Mental illness is among the most important causes of sickness, disability, and in certain age groups, premature mortality in developing countries [7]. Addressing mental health is an integral aspect of health system interventions to achieve the Millennium Development Goals (MDG) [8]. Poor and marginalized people are at higher risk for preventing attainment of MDG1, the eradication of extreme poverty and hunger [9]. The prevention of MDG 2, the achievement of universal primary education, may be related to developmental and mental disorder, and learning disability [10]. MDG 4, the reduction in child mortality, is likely associated with mental illness in pregnancy. Failure to thrive of infants $<1$ year of age is independently associated with depression of mothers in pregnancy [11].

\subsection{Global Burden of Childhood Mental Illness}

About $30 \%$ of the population globally are affected by a mental disorder and more than two-thirds of those affected do not receive needed care. With $14 \%$ of the global disease burden attributed to neuropsychiatric disorders, 1 in 17 people have a serious mental health condition [12]. Roughly, $10 \%$ to $20 \%$ of children are affected by one or more mental or behavioral problems [13], with estimates from the Western Cape region of South Africa of 17\% that have a mental disorder [14]. In conflict areas such as Mosul, Iraq the prevalence of mental illness 
can be $35 \%$ whereas only $15 \%$ to $30 \%$ of children worldwide receive the treatment they need [15] [16].

\subsection{Global Inequalities Influencing Childhood Mental Health}

According to the WHO, health inequalities are defined as "differences in health status or in the distribution of health determinants between different population groups" [16]. They deter access to care, use and outcomes of care, affecting all geographical regions whether rural or suburban; both genders, racial and ethnic background and sexual orientation. The poor are at a greater risk of developing mental illness compared to non-poor, and poverty is a determinant and consequence of poor mental health. In developing nations where resources and infrastructure are scarce, advocacy and political influence is often lacking. Families of people with mental health problems may be marginalized and limited in their ability to champion mental health issues because of the associated stigma.

\subsection{Integrating Mental Health into Primary Pediatric Care}

With a mismatch of mental health resources to those in need varying from 1 psychiatrist for every 100,000 people in much of south-east Asia, to less than one for every 1 million people in sub-Saharan Africa [16], there is a scarcity of psychiatric hospitals that are typically located in urban settings and away from family members. A key strategy for addressing inequalities in mental health care for children will be to integrate mental health within other primary care services. The reasons for integrating mental health into primary pediatric care are to make it affordable and cost-effective, promoting access and respect. Community mental health services can help reduce social stigma and discrimination by reducing the social isolation, neglect, and institutionalization of people living with mental health problems.

\subsection{Genetic Insights}

Studies of MDD in twins and families show increased heritability however, molecular studies have failed to identify susceptibility genes convincingly. Genome-wide association studies (GWAS) of single nucleotide polymorphisms (SNPs) are important in the study of the genetic basis of MDD and recurrent depressive disorder [1].

\section{Conclusion}

Childhood mental illness is a major factor of overall health that continues the lifespan of children continuing into adulthood. Population based surveys and surveillance provide much of the evidence needed to understand mental health promotion, mental illness prevention, and treatment programs in the United States. Childhood mental health disorders present serious deviations from expected cognitive, social, and emotional development, which are an important public health issue in the United States and worldwide. 


\section{Disclose}

The author has nothing to disclose.

\section{References}

[1] Younger, D.S. (2016) Epidemiology of Childhood and Adult Mental Illness. Neurologic Clinics, 34, 1023-1033. https://doi.org/10.1016/j.ncl.2016.06.010

[2] US Department of Health and Human Services (1999) Mental Health: A Report of the Surgeon General. US Department of Health and Human Services, Substance Abuse and Mental Health Services Administration, Center for Mental Health Services, National Institutes of Health, National Institute of Mental Health, Rockville.

[3] World Health Organization (2004) Promoting Mental Health: Concepts, Emerging Evidence, Practice (Summary Report). World Health Organization, Geneva, Switzerland.

[4] Perou, R., Bitsko, R.H., Blumberg, S.J., et al. (2013) Mental Health Surveillance among Children-United States, 2005-2011. MMWR Surveillance Summaries, 62, 1-3.

[5] American Psychiatric Association (2000) Diagnostic and Statistical Manual for Mental Disorders. 4th Edition, American Psychiatric Association, Washington DC.

[6] World Health Organization (1992) The ICD-10 Classification of Mental and Behavioural Disorders. Clinical Descriptions and Diagnostic Guidelines. World Health Organization, Geneva, Switzerland.

[7] Miranda, J.J. and Patel, V. (2005) Achieving the Millennium Development Goals: Does Mental Health Play a Role? PLoS Medicine, 2, 2291.

https://doi.org/10.1371/journal.pmed.0020291

[8] United Nations (2000) United Nations Millennium Declaration: Resolution Adopted by the General Assembly (No. A/RES/55/2) (8th Plenary Meeting). United Nations General Assembly, New York.

[9] Patel, V. and Kleinman, A. (2003) Poverty and Common Mental Disorders in Developing Countries. Bulletin of the World Health Organization, 81, 609-615.

[10] Patel, V. and De Souza, N. (2000) School Drop-Out. A Public Health Approach for India. National Medical Journal of India, 13, 316-318.

[11] Patel, V., Rahman, A., Jacob, K.S. and Hughes, M. (2004) Effect of Maternal Mental Health on Infant Growth in Low Income Countries. New Evidence from South Asia. BMJ, 328, 820-823. https://doi.org/10.1136/bmj.328.7443.820

[12] Kessler, R.C., Chiu, W.T., Demler, O. and Walters, E.E. (2005) Prevalence, Severity, and Comorbidity of 12-Month DSM-IV Disorders in the National Comorbidity Survey Replication. Archives of General Psychiatry, 62, 617-709. https://doi.org/10.1001/archpsyc.62.6.617

[13] Murthy, R., Bertolote, J., Epping-Jordan, J.A., et al. (2001) The World Health Report Mental Health: New Understanding New Hope. World Health Organization, Geneva.

[14] Kleintjes, S., Flisher, A., Fick, M., et al. (2006) The Prevalence of Mental Disorders among Children, Adolescents and Adults in the Western Cape, South Africa. South African Psychiatry Review, 9, 157-160. https://doi.org/10.4314/ajpsy.v9i3.30217

[15] Al-Jawadi, A.A. and Abdul-Rhman, S. (2007) Prevalence of Childhood and Early Adolescence Mental Disorders among Children Attending Primary Health Care Centers in Mosul, Iraq: A Cross-Sectional Study. BMC Public Health, 7, 274. https://doi.org/10.1186/1471-2458-7-274

[16] WHO (2005) Atlas of Child and Adolescent Mental Health Resources, Global Concerns, Implications for the Future. World Health Organization, Geneva. 
Submit or recommend next manuscript to SCIRP and we will provide best service for you:

Accepting pre-submission inquiries through Email, Facebook, LinkedIn, Twitter, etc. A wide selection of journals (inclusive of 9 subjects, more than 200 journals)

Providing 24-hour high-quality service

User-friendly online submission system

Fair and swift peer-review system

Efficient typesetting and proofreading procedure

Display of the result of downloads and visits, as well as the number of cited articles Maximum dissemination of your research work

Submit your manuscript at: http://papersubmission.scirp.org/

Or contact wijns@scirp.org 